\section{Furosemide Nebulization in Patients With COPD Exacerbation}

\section{To the Editor:}

We read with keen interest the article in the November 2013 issue by Motahar Vahedi and colleagues. ${ }^{1}$ They described furosemide as an adjunctive therapy for exacerbation of COPD. However, there are a few interesting points that need to be discussed before applying Motahar Vahedi's results in day to day practice. First, noninvasive ventilation (NIV) is the standard of care for COPD exacerbation. ${ }^{2}$ In the study by Motahar Vahedi et al the patients had respiratory acidosis, with mean $\mathrm{pH}$ of 7.29 and 7.27 in the intervention and placebo groups, respectively. That cohort was likely to benefit from NIV, but NIV was not used, ${ }^{2}$ so we argue that the patients did not get the optimum treatment for COPD exacerbation, so the adjunctive therapy was not compared with optimum therapy, and the study therefore does not provide information on the role of nebulized furosemide as adjunctive therapy in optimally treated COPD exacerbation.

Second, spirometry may be difficult to perform and inaccurate in COPD exacerbation patients in the emergency department, ${ }^{3}$ so $\mathrm{FEV}_{1}$ may not be a useful and objective measurement in this setting.

Third, all the patients were given supplemental oxygen at $0.5 \mathrm{~L} / \mathrm{min}$ for $30 \mathrm{~min}$, irrespective of their baseline oxygenation status. It is not clear from the paper whether oxygen was continued and the flow titrated to maintain the recommended oxygen saturation, 88-92\%..$^{2}$ In the absence of hypoxemia, is the routine use of oxygen useful in these patients?

Fourth, Motahar Vahedi et al excluded patients with cardiac failure; however, there was no mention of cor pulmonale, nor of whether the diagnosis of cardiac failure was based on clinical signs alone, or also on imaging. This is important because some of the benefits of furosemide may be related to its diuretic effect in the presence of cor pulmonale.

Fifth, whether the effects of furosemide vary with the severity of COPD could not be interpreted from the study. A sub-categorization of the study group according to Global Initiative for Chronic Obstructive Lung Disease (GOLD) classification would provide more information on the benefit of furosemide.
Sixth, the study did not discuss adverse effects or outcomes such as how many patients eventually required ICU admission, invasive or noninvasive ventilation, hospital stay, or mortality, nor whether these were significantly different between the 2 groups.

Vijay Hadda MD Karan Madan DM Anant Mohan MD

Irfan Ismail Ayub MD

Department of Pulmonary Medicine and Sleep Disorders

All India Institute of Medical Sciences New Delhi, India

The authors have disclosed no conflicts of interest.

DOI: $10.4187 /$ respcare. 03034

\section{REFERENCES}

1. Sheikh Motahar Vahedi H, Mahshidfar B, Rabiee H, Saadat S, Shokoohi H, Chardoli M, Rahimi-Movaghar V. The adjunctive effect of nebulized furosemide in COPD exacerbation: a randomized controlled clinical trial. Respir Care 2013;58(11):1873-1877.

2. Vestbo J, Hurd SS, Agusti AG, Jones PW, Vogelmeier C, Anzueto A, et al. Global strategy for the diagnosis, management and prevention of chronic obstructive pulmonary disease: updated 2013. Am J Respir Crit Care Med 2013;187(4):347-365.

3. Emerman CL, Lukens TW, Effron D. Physician estimation of $\mathrm{FEV}_{1}$ in acute exacerbation of COPD. Chest 1994;105(6):1709-1712.

\section{Furosemide Nebulization in Patients With COPD Exacerbation-Reply}

In Reply:

We appreciate the salient observations and comments by Hadda et al. It is our sincere hope that this response provides the necessary data and clarification to guide future applications of our study results in the care of patients with COPD.

Although we concur that noninvasive ventilation (NIV) is an important modality in the acute care of patients with COPD exacerbation, our research protocol was not intended to modify or change our institutional standard of care for the purpose of this study. Though the use of NIV in acute care is increasing, its use is still low in some countries. There have been no reports on the use of NIV for COPD exacerbations in our institution; however Ambrosino et al found that in $20-52 \%$ of hospitals in European countries, NIV was unavailable, and its utilization rate was $15-80 \%$ in patients with COPD exacerbation. ${ }^{1}$

Pursuing a randomized controlled trial methodology in our study, we hoped to minimize the impact of different treatment methods on the study outcome, and to compare and measure the effects of inhaled furosemide in each group. However, our methodology, and randomized controlled trials in general, often deal with very highly selected patients and assess care patterns that do not necessarily fit each and every institution. In addition, randomized controlled trials are usually too small and of too short duration to permit evaluation of delayed effects of the studied treatment. Therefore, integration of this practice in the clinical care of patients may require further multiinstitution investigations.

Many clinicians feel apprehensive about conducting spirometry, because of uncertainties about performing and interpreting it, and about the patient's cooperation in the tests. In our study we had concerns regarding the clinicians' technical ability to perform the test and interpret the results. We were able to execute the tests, as we reported, by providing appropriate training and extra efforts. The Global Initiative for Chronic Obstructive Lung Disease international COPD guidelines ${ }^{2}$ advises spirometry to measure lung function. We used spirometry to quantify breathlessness in our subjects, with or without the intervention.

Hypoxemia is defined as $\mathrm{P}_{\mathrm{aO}_{2}}$ of $<55 \mathrm{~mm} \mathrm{Hg}$ or oxygen saturation of $<90 \% .^{3}$ Oxygen therapy should be deliberately provided in patients with COPD. However, in patients with $\mathrm{S}_{\mathrm{pO}_{2}}$ of $<90 \%$, the oxygen should be titrated to achieve $\mathrm{S}_{\mathrm{pO}_{2}}$ of $90 \% .{ }^{4}$ In our study the mean oxygen saturations in the intervention and control groups were $84.8 \pm 9.6 \%$ and $82.8 \pm 4.7 \%$, respectively. Regarding equal treatment, we considered $0.5 \mathrm{~L} / \mathrm{min}$ oxygen for $30 \mathrm{~min}$ for all these hypoxemic patients as the standard treatment, and the decision on the continuation of oxygen therapy was made by a physician for every patient individually, according to standards of COPD treatment.

Hojat Sheikh Motahar Vahedi MD Shariati Hospital Emergency Department Tehran University of Medical Sciences Tehran, Iran 